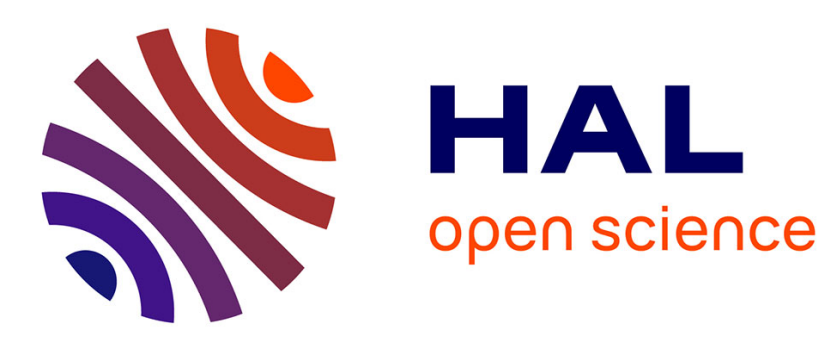

\title{
Exceptional Attributed Subgraph Mining To Understand The Olfactory Percept
}

Maëlle Moranges, Marc Plantevit, Arnaud Fournel, Moustafa Bensafi, Céline Robardet

\section{- To cite this version:}

Maëlle Moranges, Marc Plantevit, Arnaud Fournel, Moustafa Bensafi, Céline Robardet. Exceptional Attributed Subgraph Mining To Understand The Olfactory Percept. 21st International Conference on Discovery Science, Oct 2018, Limassol, Cyprus. pp.276-291. hal-01878375

\section{HAL Id: hal-01878375 \\ https://hal.science/hal-01878375}

Submitted on 21 Sep 2018

HAL is a multi-disciplinary open access archive for the deposit and dissemination of scientific research documents, whether they are published or not. The documents may come from teaching and research institutions in France or abroad, or from public or private research centers.
L'archive ouverte pluridisciplinaire HAL, est destinée au dépôt et à la diffusion de documents scientifiques de niveau recherche, publiés ou non, émanant des établissements d'enseignement et de recherche français ou étrangers, des laboratoires publics ou privés. 


\title{
Exceptional Attributed Subgraph Mining To Understand The Olfactory Percept
}

\author{
Maëlle Moranges ${ }^{1,2}$, Marc Plantevit ${ }^{1,3}$, Arnaud Fournel ${ }^{1,4}$, Moustafa \\ Bensafi $^{1,4}$, and Céline Robardet ${ }^{1,2}$ \\ 1 Université de Lyon, France \\ 2 INSA Lyon, LIRIS, CNRS UMR5205, France \\ 3 Université Lyon 1, LIRIS, CNRS UMR5205, France \\ 4 CNRS, CRNL, UMR5292, INSERM U1028, France
}

\begin{abstract}
Human olfactory perception is a complex phenomenon whose neural mechanisms are still largely unknown and novel methods are needed to better understand it. Methodological issues that prevent such understanding are: (1) to be comparable, individual cerebral images have to be transformed in order to fit a template brain, leading to a spatial imprecision that has to be taken into account in the analysis; $(2)$ we have to deal with inter-individual variability of the hemodynamic signal from fMRI images which render comparisons of individual raw data difficult. The aim of the present paper was to overcome these issues. To this end, we developed a methodology based on discovering exceptional attributed subgraphs which enabled extracting invariants from fMRI data of a sample of individuals breathing different odorant molecules.Four attributed graph models were proposed that differ in how they report the hemodynamic activity measured in each voxel by associating varied attributes to the vertices of the graph. An extensive empirical study is presented that compares the ability of each modeling to uncover some brain areas that are of interest for the neuroscientists.
\end{abstract}

\section{Introduction}

Olfaction is a chemical sense whose functions is to detect the presence of odorous substances present in the environment in order to modulate appetitive, defensive and social behaviors $[26,6]$. Olfactory deficits are a common symptom of neurodegenerative or psychiatric disorders and clinical research proposed that olfaction could have great potential as an early biomarker of disease [2,25] for example using neuroimaging to investigate the breakdown of structural connectivity profile of the primary olfactory networks. On a fundamental level, whereas olfaction has received much attention over the last decades, human olfactory perception is a complex phenomenon whose mechanisms are still largely unknown.

Neuroscientific investigations revealed that perception of odors results from the interaction between volatile molecules (described by multiple physicochemical descriptors) and olfactory receptors located in the nasal cavity. Once the interaction is done, a neural signal is then transmitted to central areas of the 
brain to generate a percept called "odor" that is often accompanied by a strong hedonic or emotional tone (either pleasant or unpleasant). Understanding the link between odor (hedonic) perception and its underlying brain activity is an important challenge in the field. Although past brain imaging studies revealed that the brain activation in response to smells is distributed and can represent different attributes of odor perception (from perception of irritation to intensity or hedonic valence) $[10,13,16]$, there is clear need to develop new brain imaging analysis techniques in order to (i) take into account the large variability across individuals in terms odor perception and brain activation, and (ii) refine the network and understand for instance how different sub-parts of a given area are involved in the processing of pleasant and unpleasant odors. This will be the main aims of the present paper.

The most popular method to acquire brain imaging data in humans is called functional Magnetic Resonance Imaging or fMRI. An important issue when performing inter-individual analysis on fMRI data is that each individual image is transformed in order to fit and map onto a template (so that comparison across participants can be made on a unique model of the brain) [11]. Therefore, voxel (i.e., 3d pixel) mapping from the individual to the template may be imprecise, and looking for voxels that have a strong hemodynamic response for all individuals can be unsuccessful. One solution to circumvent this problem is thus to take into account this imprecision by looking for areas whose voxels - although imprecise - have specific hemodynamic response to some odors for a large proportions of individuals compare to the rest of the brain. To achieve this goal, we propose to model fMRI images as an attributed graph where the vertices are the voxels (brain unit), the edges encode the adjacency relationship, and vertex attributes stand for the hemodynamic response to an odor. We propose to analyze such a graph with CENERGETiCs [4] which makes possible to identify brain areas with exceptional hemodynamic response in some experimental settings.

Commonly, in order to demonstrate a functional activity of a given voxel, neuroscientists make use of general linear model coupled with massive univariate statistics [12] whereby the mean activity a voxel is compared in a "test condition" (e.g. when participants are asked to breathe odors) and a "control" condition (e.g. when participants are asked to breathe non-odorized air). The statistical comparison is usually performed using a Student $t$-test. However, this type of comparison presents some weaknesses when trying to take into account inter individual variability. Figure 1 illustrates this issue. The distributions of the $t$ values associated to the hemodynamic responses that come from the fMRI of two individuals smelling the same odor (EUG, the Eugenol molecule that smells like cloves) are represented. If we consider that a voxel is activated when $t$ is greater than 1.96, that corresponds to an error of type 1 of $5 \%$, in one case there are almost $5 \%$ of the voxels that are activated whereas in the other case this number is lower than $10^{-2} \%$. Thus, if we look for the invariants between individuals for the same odor, there is a good chance that they do not exist.

We propose different ways to evaluate the level of activation of a voxel, based on normalized average values, ranks, t-test or pairwise comparisons. In the first 

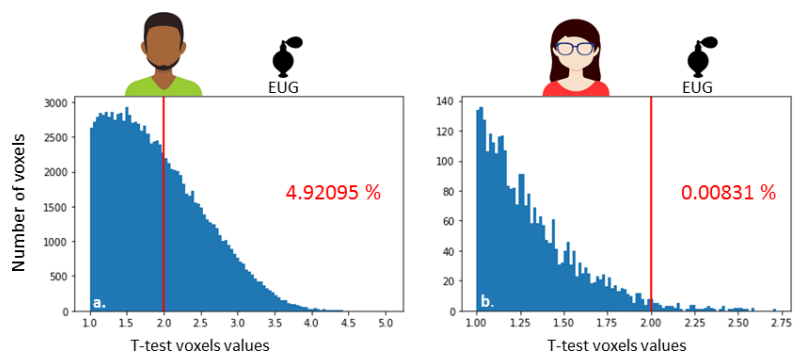

Fig. 1. Distribution of the $t$-test values associated to the voxels of two individuals smelling EUG odor.

approach, the level of activation of a voxel by an odor is evaluated by the average value of the hemodynamic response of this odor. The second proposal captures the average, for all individuals, of the rank of the odor-related response among all the responses of an individual when perceiving different odors. In the third model, the odor activates the voxel if its hemodynamic response is statistically significant according to the Student's $t$-test. In the fourth model, the attributes are pairwise comparisons of odor responses (e.g. odor $1>$ odor2) and their values are the number of individuals for which this comparison holds.

Exceptional subgraphs on fMRI data are presented in Section 2, as well as the attributed graphs built to model the brain activation during olfactory perception. Such patterns can be mined using CENERgETICS, an algorithm designed to discover connected subgraphs with over-represented and/or under-represented attributes, that was developed to analyze urban data. CENERGETiCs is applied on these graphs and the obtained results are compared through an extensive empirical study in Section 3. Related work is reviewed in Section 4 and concluding remarks are given in Section 5.

\section{Mining Exceptional Subgraphs For Olfactory Percept Analysis}

We propose to use pattern mining techniques to identify relationships between odor perception and brain areas. The data we analyze come from a neuroscience experiment measuring hemodynamic responses when perceiving different odors using fMRI. $p$ individuals participated in the study and each of them inhaled $q$ different odors $t$ times. During each olfactory trial, a brain volume is acquired. Each image reflects the hemodynamic response in each of 3 millimeters cubed unit of the brain, hereafter called voxel. The hemodynamic response function is then modeled as regressors that render hemodynamic activity [23]. Let us denote by $X_{k}(v, i, j)$ the level of activity measured in the voxel $v$ for individual $i=1 \ldots p$ while smelling an odor $j=1 \ldots q$, at time $k=1 \ldots t$, and $A_{k}(v, i)$ the activity measured while individual $i$ is breathing air. The specific activity value 
of an odor perception is obtained by the average difference of $X$ and $A$ :

$$
M(v, i, j)=\frac{\sum_{k}\left(X_{k}(v, i, j)-A_{k}(v, i)\right)}{t}
$$

However, the measure $M$ is not bounded, taking positive and negative values, with its intensity depending on the sensitivity of the participant. To be able to compare different individuals, it is usual in neuroscience to normalize the value $M$ that is tranformed into a statistical Student t-value:

$$
M^{\prime}(v, i, j)=\frac{M(v, i, j)}{\frac{\sigma(X-A)}{\sqrt{t}}}
$$

with $\sigma(X-A)$ the standard deviation of the measures $X-A$ over $k$.

In an inter-individual analysis, we are interested in voxel areas (1) that are activated by a given odor for most of the individuals and (2) whose activation level is much higher than that observed in other areas of the brain. To identify such patterns, we propose to mine exceptional subgraphs in an attributed graph which models the brain activation when the subject is stimulated by an odor.

\subsection{Mining Activated Areas In The Brain}

Brain activity can be modeled as a vertex attributed graph whose vertices $V$ represent voxels and edges $E$ connect adjacent voxels. A set of attribute value pairs $P$ is associated to each vertex and describes the activity of the corresponding voxel. The attributes are denoted $\mathcal{A}$ and take their value $x$ in $\mathbb{R}$ :

$$
P: V \rightarrow\{(a, x) \mid a \in \mathcal{A}, x \in \mathbb{R}\}
$$

Our objective is to identify brain areas whose attribute value pairs distinguish them from the rest of the brain. To this end, we propose to discover connected subgraphs associated to exceptional attribute value pairs. An attribute value pair is considered as exceptional for a subgraph if it has a much higher value in its vertices than in the remaining of the graph. Hence, an exceptional attributed subgraph is defined as a pair $(S, K)$ with $S \subseteq V$, a subset of vertices that induces the subgraph $G[S]$, and $K \subseteq \mathcal{A}$ a subset of attributes whose values are exceptional for this subgraph. This is evaluated by the weighted relative accuracy defined as:

$$
\operatorname{WRAcc}(S, K)=\frac{\operatorname{sum}(S, \mathcal{A})}{\operatorname{sum}(V, \mathcal{A})} \times\left(\frac{\operatorname{sum}(S, K)}{\operatorname{sum}(S, \mathcal{A})}-\frac{\operatorname{sum}(V, K)}{\operatorname{sum}(V, \mathcal{A})}\right)
$$

with $\operatorname{sum}(S, K)=\sum_{v \in S} \sum_{(a, x) \in P(v), a \in K} x$.

Definition 1 (Exceptional attributed subgraph). Given an attributed graph $G=(V, E, \mathcal{A}, P)$ and two thresholds minV and $\delta$, an Exceptional attributed sub$\operatorname{graph}(S, K)$ is such that (1) $|S| \geq \min V$, (2) $G[S]$ is connected, (3) $W R A c c(S, K) \geq$ $\delta$ and (4) $\forall v \in S, \forall p \in K, W R A c c(v, p)>0$. 
Condition (1) ensures that patterns involve enough vertices to be of interest. Condition (2) preserves the notion of areas and avoid discontinuity. Condition (3) assesses the exceptionality of the attributes, while condition (4) enforces the subgraph to be cohesive. Such patterns can be mined using the algorithm presented in [4], originally designed to exhibit the predominant activities and their associated urban areas in graphs that model urban areas. The algorithm, named Cenergetics, mines exceptional subgraph in attributed graphs.

Exceptional attributed subgraph definition can be extended to also catch attributes whose values are exceptionally lower for the subgraph than for the rest of the graph. CENERGETICS enables the possibility of discovering subgraphs with both exceptionally over- or under-represented attributes. In this section, we consider over-represented attribute values. The case of under-represented attributes is discussed in the empirical study.

Several attributed graphs can be constructed based on fMRI data. They differ by the attribute value pairs associated to the vertices, that is to say, by the attribute and by the value associated to them.

\subsection{Attributed Graphs That Model Olfactory Perception}

The attribute value pairs $P$ of the graph reflects the strength of the hemodynamic response of the corresponding voxel when perceiving the odors. The attributes $\mathcal{A}$ can be the odor names, but also another characteristics such as chemical properties or the feelings felt during the perception (for instance their pleasant or unpleasant character). We denote by $\pi$ the characteristic used to describe the odor (i.e. an injective function from odors to a set of labels). The attributes of $\mathcal{A}$ can also be pairs of odors to characterize voxels with pairwise inequalities.

The value of each attribute results of the aggregation of the measurements $M$ obtained for different individuals. Since these measurements may contains errors, which may come from the material used, but also from the brain activity of the participant during the experiment (stress, thoughts), we consider below different ways of aggregating the data. These different approaches attempt to overcome this problem and will be experimentally compared.

Mean of the values: A voxel activation can be characterized by the mean of the values:

$$
(\pi[j], x) \in P(v) \text { with } x=\frac{\sum_{i} M^{\prime}(v, i, j)}{p}
$$

To limit the effect of high inter-individual variability it can be preferable not to consider the measure $M^{\prime}$ as an interval scale, but to downgrade the type of measurement scale and only consider the ranks.

Average rank: The voxel activation is evaluated by the average rank of the odor in the individual perceptions:

$$
(\pi[j], x) \in P(v) \text { with } x=\frac{\sum_{i} \operatorname{rank}(v, i, j)}{p}
$$

with $\operatorname{rank}(v, i, j)=\left|\left\{\ell=1 \ldots q \mid M^{\prime}(v, i, \ell) \leq M^{\prime}(v, i, j)\right\}\right|$. 
t-test based approach: We can also downgrade the measure to consider it as a nominal variable. The discretization can be obtained thanks to a $t$-test assesses whether a voxel is activated or not. For a voxel $v$, an individual $i$ and an odor $j$, if $M^{\prime}(v, i, j)$ is greater than the critical value with $d f=t-1$ of the student distribution (given the confidence level $\alpha=0.05$ ), then the hemodynamic response is considered to be different from the one observed while breathing air and the voxel is esteemed activated:

$$
(\pi[j], x) \in P(v) \text { with } x=\left|\left\{i=1 \ldots p \mid\left(T_{0.05}<M^{\prime}(v, i, j)\right)\right\}\right|
$$

Approach based on pairwise inequality: We propose another setting based on the pairwise comparison of the hemodynamic responses. The vertex attributes are pairs of odors $\left(o_{1}, o_{2}\right)$ and their value is the number of individuals who have a higher value while smelling $o_{1}$ than when smelling $o_{2}$. Thereby, $q \times(q-1)$ attributes (the number of pairs of odors) are associated to each vertex $v$ of the graph and their values are:

$$
\left(\left(\pi\left[o_{1}\right], \pi\left[o_{2}\right]\right), x\right) \in P(v) \text { with } x=\left|\left\{i=1 \ldots p \mid M^{\prime}\left(v, i, o_{1}\right)>M^{\prime}\left(v, i, o_{2}\right)\right\}\right|
$$

\section{$3 \quad$ Empirical Study}

In this section, we report our experimental results. These experiments aim to compare the different ways to build an attributed graph that are described in the previous section. Especially, we want to identify which ones are the most promising to identify exceptional subgraphs and how the related patterns make sense. To this end, we study the main characteristics of the discovered patterns for each modeling. In our experiments, 14 individuals smelled 6 odorants $^{1} 10$ times $(p=14, q=6$ and $k=10)$.

As mentioned in the previous section, exceptional attributed subgraph definition can be extended to make possible the discovery of subgraphs whose attribute values are lower than what observed on the rest of the graphs. For mean and rank modelings, we just adapted the WRAcc measure to catch under-represented attributes [4]. In the pairwise inequality based modeling, we consider all pairs of attributes, so it is not necessary to consider under-represented attribute values. For the $t$-test based method, the under-representedness is captured by the lower tail of the $t$-test distribution $(\alpha=0.05)$. In the following, the over-represented attributes are called positive and the under-represented ones are named negative.

Experiments were carried out on an Intel Core i7-4770 3.40GHz machine with 8GB RAM. Applied on the whole brain (902629 voxels), Cenergetics takes at most 7 minutes to discover the exceptional subgraphs. However, in the following, we focus on the piriform cortex, an area made of 662 voxels known as the first olfactory area that receives information of the olfactory bulb. Here, we aim to understand how odors and their perceptual properties (hedonic) are processed in this area compared to the other brain areas.

\footnotetext{
${ }^{1}$ The odorant names are: $3 \mathrm{Hex}, \mathrm{ACE}, \mathrm{DEC}, \mathrm{EUG}, \mathrm{HEP}, \mathrm{MAN}$
} 
For this study, the parameters of CENERGETICS are such that the computed exceptional subgraphs contain at least one vertex and have a WRAcc value greater than 0.004. Extractions take at most $201 \mathrm{~ms}$ regardless of the considered models. The number of patterns are as follows: 555 for Mean, 238 for Rank, 118 for $t$-test and 803 for pairwise modelings.

This empirical study aims to answer the following questions: (a) Are the collections of exceptional subgraphs obtained with the 4 modelings different? (distributions) (b) Do the different modeling capture the same phenomena? (same attributes, similar areas) (c) What about considering odor characteristics? (hedonic values) (d) Do the discovered subgraphs make sense? What kind of insights can they provide to neuroscientists?

To this end, we first study the main characteristics of the exceptional attributed subgraphs obtained by each modeling. We then provide a detailed crossed-analysis of the top 3 patterns of the four collections. Finally, we consider other attributes related to the odorants to discuss the potential of each modeling. We also provide neuroscientists' feedback on these patterns. Additional results are provided as supplementary material ${ }^{2}$.

\subsection{Comparison Of Patterns Obtained From The Different Models}

Fig. 2 reports the distribution of the patterns according to their WRAcc value. The distributions of the four approaches are similar. Nevertheless, $t$-test and mean based modelings retrieve patterns with the highest WRAcc values. The pairwise inequality based modeling provides patterns with lower WRAcc values. This can be due to the total number of attributes that is larger for the pairwise model (30) than for the other approaches (12).

Similarly, we show the distribution of patterns according to the number of vertices they contain in Fig. 3. Patterns discovered by the $t$-test based approach contain less vertices than the ones retrieved by the other modelings. One possible consequence of this observation is a greater risk to provide false positive patterns

${ }^{2}$ goo.gl/ppJFEX
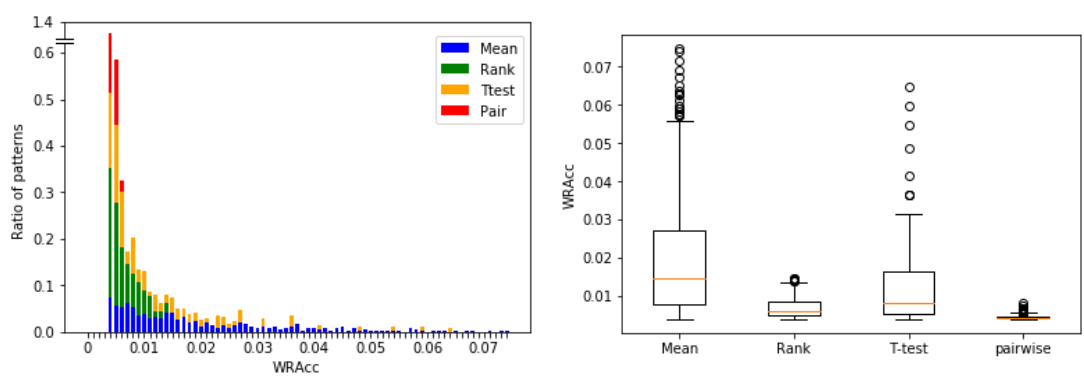

Fig. 2. Distributions of patterns with respect to their WRAcc value for each approach (left). Statistical characteristics of these distributions (right). 

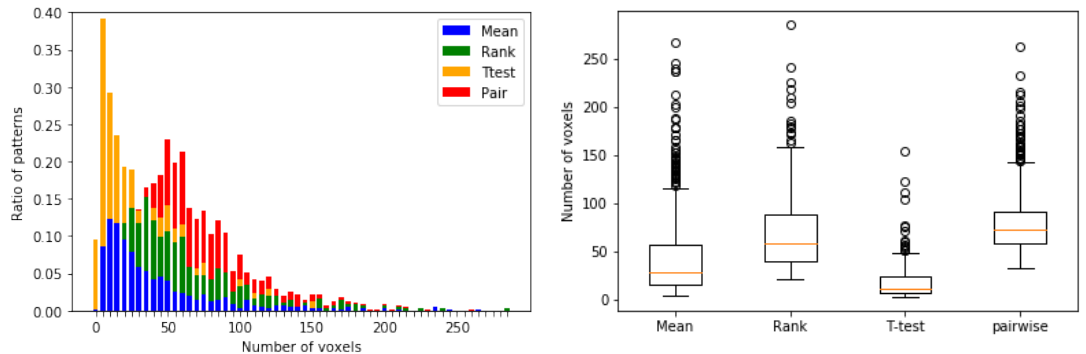

Fig. 3. Distribution of patterns with respect to their number of voxels for each approach (left). Statistical characteristics of these distributions (right).

to the end-user. Other methods have a smoother distribution, methods rank and pairwise giving the biggest patterns.

We also study the distribution of patterns with respect to their number of attributes. As the maximum number of attributes is different for the four modelings, we normalize the observed number of attributes by dividing the observed number of attributes by the maximal possible number of attributes in a pattern - 15 for the pairwise based approach and 6 for the others (opposite attributes cannot appear in a same pattern). Results are given in Fig. 4. The patterns discovered with $t$-test based approach have, in general, a lower number of attributes than the patterns obtained with other approaches. When normalized, the distribution of the patterns discovered with the pairwise inequality based approach is greater than the others.

Fig. 5 reports the distributions of patterns according to the number of the individuals that participate to the patterns. In reality, individuals do not directly participate to a pattern, but it is the hemodynamic response measured by their fMRI on the voxels of the pattern $(S, K)$ that indirectly associates an individual to it. For the $t$-test modeling, an individual $i$ satisfies a voxel $v \in S$
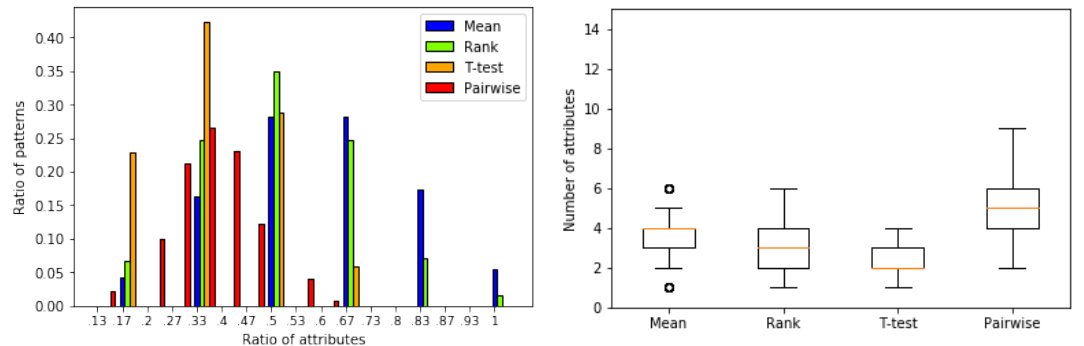

Fig. 4. Distribution of patterns with respect to their number of attributes for each approach (left). Statistical characteristics of these distributions (right). 

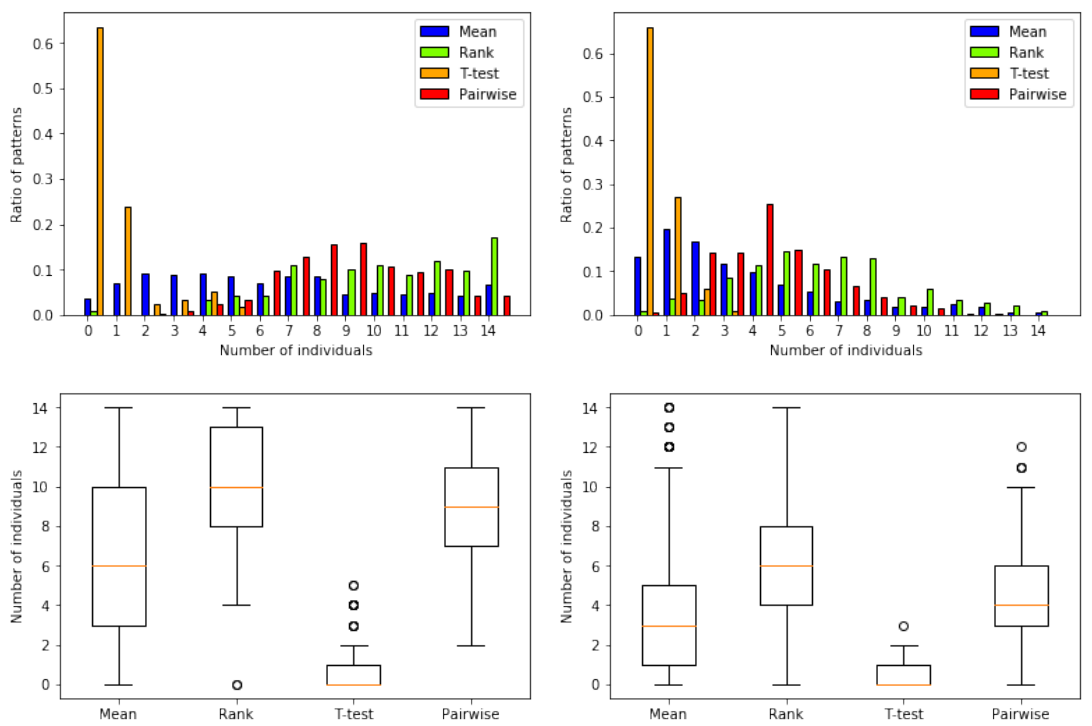

Fig. 5. Distribution of patterns with respect to the number of individuals that fulfill all the attributes on at least 1 voxel of the pattern (left) and on at least $20 \%$ of the voxels (right).

if $\forall j \in K, T_{0.05}<M^{\prime}(v, i, j)$ (for positive attributes) or $T_{0.05}>-M^{\prime}(v, i, j)$ (for negative attributes). For the pairwise modeling, an individual $i$ satisfies a voxel $v \in S$ if $\forall\left(o_{1}, o_{2}\right) \in K, M^{\prime}\left(v, i, o_{1}\right)>M^{\prime}\left(v, i, o_{2}\right)$. For the mean and the rank based approaches, an individual $i$ is considered to satisfies a voxel $v \in S$ if $\forall j \in K, M^{\prime}(v, i, j)$ is higher (for positive attributes) or lower (for negative attributes) than the mean (resp. the mean rank) over all the vertices of the graph. As none of the individuals satisfy all the voxels of a pattern, we consider two cases: one where individuals participate to pattern when they satisfy at least one of its voxels, and another one where they have to satisfy at least $20 \%$ of the pattern voxels. Doing this, we observe that the number of individuals that participate to the patterns is much lower for the $t$-test based modeling than for the other approaches.

The study of the previous distributions leads to some partial conclusions. Patterns discovered by the $t$-test based approach generally contain less vertices and attributes and are supported by fewer individuals than the other approaches. On the contrary, patterns discovered thanks to modelings that take into account the ranks of the hemodynamic responses (i.e., the rank and the pairwise inequality modelings) involve more vertices, attributes and are supported by more individuals. To discover some inter-individual invariant, $t$-test based method seems to be less suited than methods that takes into account ranks. This is what it is further investigated in the following qualitative study. 


\subsection{Qualitative Comparison Of The Top-3 Patterns}

The top 3 patterns according to the WRAcc measure discovered by the 4 approaches are reported in Table 1. Notice that these patterns are obtained after a post-processing that ensures diversified results by constraining the overlap between the top 3 patterns to be lower than $30 \%$ [4]. The notation " $o_{1}<o_{2}$ " is used to say that the hemodymamic response of the odor $o_{1}$ is lower that the hemodymamic response of the odor $o_{2}$. The numbers of individuals reported in Table 1 are the numbers of individuals who participate to at least one voxel of the patterns. Fig. 7 shows, for each pattern, the distribution of the percentage of its voxels that are satisfied by the individuals. To evaluate how these patterns overlap each others, we compute the Jaccard similarity of their set of vertices. Fig. 6 reports these values when they are greater than 0.30 . We can observe that: (a) The best 3 patterns respectively found for the mean, rank and pairwise approaches match each others. They have a strong Jaccard index (between 0.42 and 0.67 ). Both the mean and the rank based approaches give us exactly the same pieces of information in term attributes. The pairwise based approach is in agreement with these patterns but it provides additional insights. For the first pattern, for instance, three other odorants (3Hex, HEP and MAN) have also a hemodynamic response greater than the ACE's one. (b) The best pattern discovered by the $t$-test based approach does not match any other patterns (Jaccard index lower than 0.3 ). Furthermore, only 4 individuals support at least one vertice and only 2 support at least $10 \%$ of the vertices. The second one has a small overlap with the third patterns of both mean and rank approaches $(0.35)$ but concerns other odorants. The third $t$-test based pattern overlaps with the first patterns of the other approaches. Even though it is not in contradiction with the pairwise approach, it concerns different odors compared to the other two methods. (c) The second pattern obtained thanks to the pairwise based modeling overlaps with the third pattern of both mean and rank approaches. It provides additional information compared to these patterns as $D E C<A C E$ and $3 H e x<A C E$ relationships are also present in the pattern.

To conclude, the pairwise based approach gives more pieces of information than the other ones and patterns are better supported by individuals.

\subsection{Patterns Based On Other Odor Characteristics}

The discovery of exceptional attributed subgraphs in which the attributes are the odorants leads to the identification of areas of interest for the neuroscientists. However, the odorant properties are not taken into account in the analysis and thus their interpretation requires much effort. Neuroscientists aim to find links between brain areas and some odorant attributes, especially their hedonic perception during the fMRI measurement. During the experiment, the subjects must express a hedonic judgment regarding the breathed smell and say whether it is pleasant, unpleasant or neutral. In Fig. 8, the distribution of pleasant/neutral/unpleasant odorants in the patterns discovered by the four methods is reported. There is no pattern capturing only odorants that are all perceived 


\begin{tabular}{|l|l|c||c||c||c|}
\hline & Mean & Rank & t-test & Pairwise \\
\hline 1 & positive attributes & $D E C, E U G$ & $D E C, E U G$ & $H E P$ & $A C E<3 H e x, D E C, E U G, H E P, M A N$ \\
negative attributes & $A C E$ & $A C E$ & - & - \\
number of voxels & 138 & 125 & 104 & 117 \\
number of individuals & 12 & 12 & 4 & 12 \\
\hline \multirow{2}{*}{2} & $A C E$ & $A C E$ & $3 H e x$ & $3 H e x, D E C, H E P, E U G<A C E$ \\
positive attributes & negative attributes & $E U G, H E P$ & $E U G, H E P$ & - & - \\
number of voxels & 134 & 122 & 154 & 151 \\
number of individuals & 7 & 10 & 4 & 12 \\
\hline \multirow{2}{*}{3} & $A C E$ & $A C E$ & $M A N$ & $D E C<3 H e x, M A N, A C E$ \\
positive attributes & & & & $3 H e x<A C E$ \\
negative attributes & $D E C$ & $D E C$ & - & - \\
number of voxels & 178 & 173 & 123 & 119 \\
number of individuals & 12 & 14 & 5 & 14 \\
\hline
\end{tabular}

Table 1. Top 3 patterns for each modeling (see supplementary material for their visualization).
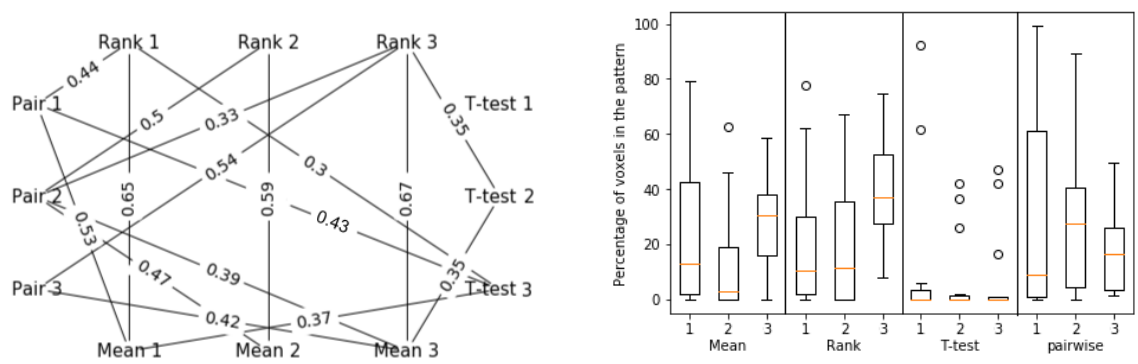

Fig. 6. Graph of the Jaccard similarities Fig. 7. Distribution of the percentage of between top 3 patterns. voxels that are satisfied by each individual for the top 3 patterns.

as pleasant (or unpleasant) by a large proportion of individuals. We then consider hedonicity as an attribute and perfom new extractions with CEnERGETICS considering the different modelings.

We enforce syntactic constraints to focus on patterns that are of interest for the neuroscientists. For the mean, rank and $t$-test based approaches, we search patterns verifying one of these conditions: (a) the hemodynamic response of odorant perceived as neutral is higher than those perceived as pleasant and unpleasant; (b) the hemodynamic response of odorant perceived as neutral is lower than those perceived as pleasant and unpleasant; (c) the hemodynamic response of odorant perceived as pleasant is higher than those perceived as unpleasant; and (d) the hemodynamic response of odorant perceived as unpleasant is higher than those perceived as pleasant. For the pairwise approach, we look for patterns whose attributes describe order between pleasant and unpleasant ${ }^{3}$.

\footnotetext{
${ }^{3}$ I.e., neutral $>$ pleasant, unpleasant, or neutral $<$ pleasant, unpleasant, or pleasant $>$ neutral $>$ unpleasant, or unpleasant $>$ neutral $>$ pleasant.
} 

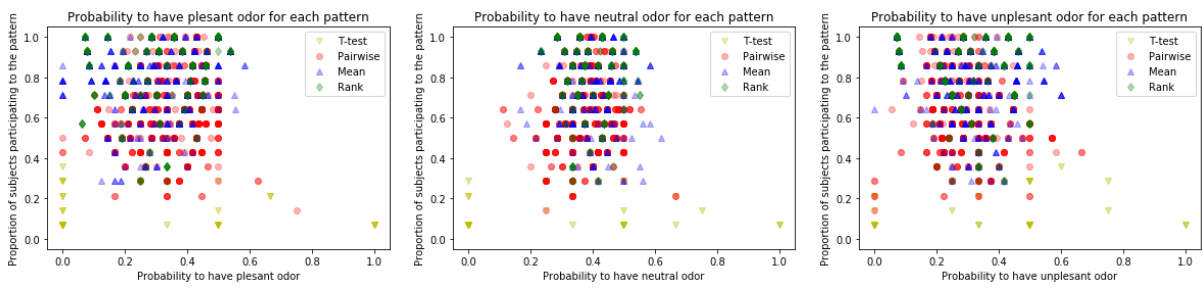

Fig. 8. Percentage of pleasantness of the dominant odors for each number of individual participating to the pattern.

Cenergetics takes less than $32 \mathrm{~ms}$ to extract the patterns for each approach. The top 5 patterns w.r.t. WRAcc measure for each method are reported in Table 2 and their brain visualization is given in Fig. 9. The patterns discovered by the $t$-test based approach are too small to be analyzed (only 1 to 5 voxels) and visualized. Patterns discovered by the different methods overlap. Those that have a Jaccard similarity greater than 0.3 (see supplementary material for more details) capture similar information (e.g. syntactic constraints). Some of these patterns highlight some areas in which polarized hedonic values have a different distribution than neutral hedonic value. This confirms neuroscientists' priors.

Indeed, the fact that the most emotional odors (pleasant and unpleasant) (blue, red and green patterns in Fig. 9) are more represented in the posterior part of the piriform cortex whereas responses to neutral odors (cyan patterns in Fig. 9: $R 4$ and $P 3)$ are more localized anteriorly within the piriform cortex is consistent with previous findings in Neuroscience $[13,16]$ showing that the posterior part of the piriform cortex represent salient perceptual experience of smells. Note that this posterior area of the piriform cortex is at the neighborhood of another area known to be involved in emotional processing, namely the amygdala.

\begin{tabular}{|c|c|c|c|c|c|}
\hline & & Mean & Rank & $t$-test & Pairwise \\
\hline \multirow{3}{*}{1} & positive attributes & $U$ & $U$ & $P$ & $N<P, U$ \\
\hline & negative attributes & $P$ & $P$ & $U$ & 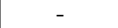 \\
\hline & number of voxels & 134 & 143 & 5 & 43 \\
\hline \multirow{3}{*}{2} & positive attributes & $P$ & $P$ & $N$ & $P<N<U$ \\
\hline & negative attributes & $U$ & $U$ & $P, U$ & _ \\
\hline & number of voxels & 137 & 150 & 4 & 44 \\
\hline & positive attributes & $P, U$ & $U$ & $N$ & $P, U<N$ \\
\hline 3 & negative attributes & $N$ & $P$ & $P, U$ & - \\
\hline & number of voxels & 58 & 66 & 1 & 61 \\
\hline & positive attributes & $P$ & $N$ & $P$ & $U<N<P$ \\
\hline \multirow{2}{*}{4} & negative attributes & $U$ & $P, U$ & $U$ & - \\
\hline & number of voxels & 64 & 28 & 1 & 41 \\
\hline & positive attributes & $P, U$ & $P, U$ & $P$ & $N<P, U$ \\
\hline \multirow[t]{2}{*}{5} & negative attributes & $N$ & $N$ & $U$ & - \\
\hline & number of voxels & 25 & 24 & 1 & 62 \\
\hline
\end{tabular}

Table 2. Top 5 patterns with the hedonic attributes : unpleasant (U), pleasant (P) and neutral $(\mathrm{N})$. 

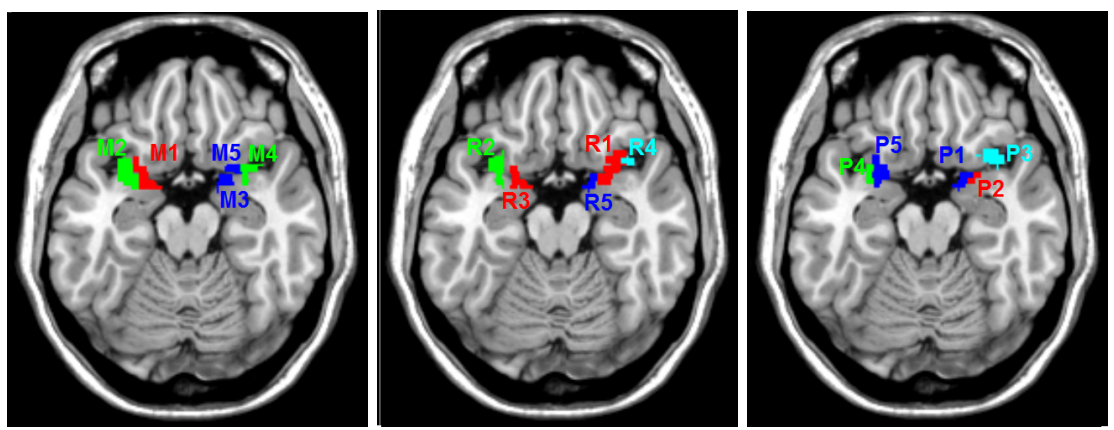

Fig. 9. Upper view of the top 5 patterns satisfying the syntactic constraints for each approach. Patterns of mean based approach (left), patterns of rank based approach (center) and patterns of pairwise inequality based approach (right). The color red represents the unpleasant pattern, green the pleasant pattern, cyan the pattern where the neutral odors are higher than the pleasant and the unpleasant odors and the blue patterns where the neutral odors are lower than the pleasant and the unpleasant odors.

The mean, rank and pairwise based modelings find similar information, which improves the neuroscientists' confidence in these findings. Furthermore, the pairwise based modeling conveys more information to the neuroscientists than the two others. This approach is promising and could be used on other odor attributes to potentially formulating new hypotheses on the olfactory percept in neuroscience.

\section{Related Work}

Scientists have always seen Exploratory Data Analysis (EDA) as an important research area since its introduction [27]. Among the various EDA techniques that aim to maximize insight into datasets and uncover underlying structures, Subgroup Discovery (SD) $[18,28]$ is a generic data mining task concerned with finding regions in the data that stand out with respect to a given target. Many other data mining tasks have similar goals as SD, e.g., emerging patterns [7], contrast sets [3]. However, among these different tasks, SD is known as the most generic one, especially SD is agnostic of the data and the pattern domain. For instance, subgroups can be defined with conjunction of conditions on symbolic [19] or numeric attributes [15, 1] as well as sequences [14]. Furthermore, the single target can be discrete or numeric [22]. Exceptional Model Mining (EMM) [21], while sharing the same exploration space (i.e., the description space), extends SD by offering the possibility to handle complex targets, e.g., several discrete attributes[20,9], two numeric targets [8] and preferences [24].

Our method is rooted in the SD/EMM framework. Nevertheless, the problem we tackle cannot be directly addressed with an instance of SD/EMM. Indeed a target space is provided instead of explicit targets. Dynamic EMM/SD (i.e., EMM/SD with a non-fixed model) has been recently investigated for different 
aims. Bosc et al. [5] propose a method to handle multi-label data where the number of labels per objects is much lower than the total number of labels which prevent the use of usual EMM model. Other dynamic EMM approaches aim to discover exceptional attributed sub-graphs $[17,4]$. Notice that in these works, exceptional subgraphs must also fulfill a structural constraint (e.g., connectivity). In this paper, we demonstrate that exceptional attributed sub-graph discovery is promising to provide new insights in neuroscience.

\section{Conclusion}

In this paper, we introduced a new way to analyze fMRI data in order to better understand olfactory perception at the cerebral level. To this end, we modelled fMRI data as an attributed graph whose vertices depict voxels and attributes encode the hemodynamic response related to a series of odorant molecules. We defined four different ways to analyze hemodynamic activity within the studied voxels. Exceptional attributed graphs were then discovered using CEnergetICS algorithm. A thorough empirical study compared the different models. The strength of such an approach lies in its ability to be more robust to spatial imprecision and inter-individual variability than classical fMRI analysis approaches: here, the pairwise inequality attribute based modeling seems to be the most promising approach. It makes possible the discovery of areas of interest supported by many individuals while conveying much more semantics than other models. This paper is the first attempt to apply attributed graph analysis to understand the olfactory perception and their neural underpinnings. It opens up several avenues for further research including definition of new pattern domains to fully take into account the brain specificity as well as prior knowledge.

\section{References}

1. Atzmüller, M., Puppe, F.: Sd-map - A fast algorithm for exhaustive subgroup discovery. In: ECMLPKDD. pp. 6-17 (2006)

2. Baba, T., Kikuchi, A., Hirayama, K., Nishio, Y., Hosokai, Y., Kanno, S., Hasegawa, T., Sugeno, N., Konno, M., Suzuki, K., et al.: Severe olfactory dysfunction is a prodromal symptom of dementia associated with parkinson's disease: a 3 year longitudinal study. Brain 135(1), 161-169 (2012)

3. Bay, S.D., Pazzani, M.J.: Detecting group differences: Mining contrast sets. Data mining and knowledge discovery 5(3), 213-246 (2001)

4. Bendimerad, A.A., Plantevit, M., Robardet, C.: Mining exceptional closed patterns in attributed graphs. Knowl. Inf. Syst. 56(1), 1-25 (2018)

5. Bosc, G., Golebiowski, J., Bensafi, M., Robardet, C., Plantevit, M., Boulicaut, J.F., Kaytoue, M.: Local subgroup discovery for eliciting and understanding new structure-odor relationships. In: DS. pp. 19-34. Springer (2016)

6. Croy, I., Bojanowski, V., Hummel, T.: Men without a sense of smell exhibit a strongly reduced number of sexual relationships, women exhibit reduced partnership security-a reanalysis of previously published data. Biological psychology 92(2), 292-294 (2013) 
7. Dong, G., Li, J.: Efficient mining of emerging patterns: Discovering trends and differences. In: KDD. pp. 43-52. ACM (1999)

8. Downar, L., Duivesteijn, W.: Exceptionally monotone models - the rank correlation model class for exceptional model mining. Knowledge and Information Systems 51(2), 369-394 (May 2017)

9. Duivesteijn, W., Feelders, A.J., Knobbe, A.: Exceptional model mining. Data Mining and Knowledge Discovery 30(1), 47-98 (2016)

10. Fournel, A., Ferdenzi, C., Sezille, C., Rouby, C., Bensafi, M.: Multidimensional representation of odors in the human olfactory cortex. Human Brain Mapping 37, 2161-2172 (03 2016)

11. Friston, K., Ashburner, J., Frith, C.D., Poline, J.B., Heather, J.D., Frackowiak, R.S., et al.: Spatial registration and normalization of images. Human brain mapping 3(3), 165-189 (1995)

12. Friston, K.J., Holmes, A.P., Worsley, K.J., Poline, J.P., Frith, C.D., Frackowiak, R.S.: Statistical parametric maps in functional imaging: a general linear approach. Human brain mapping 2(4), 189-210 (1994)

13. Gottfried, J.A., Winston, J.S., Dolan, R.J.: Dissociable codes of odor quality and odorant structure in human piriform cortex. Neuron 49(3), 467-479 (2006)

14. Grosskreutz, H., Lang, B., Trabold, D.: A relevance criterion for sequential patterns. In: ECMLPKDD. pp. 369-384 (2013)

15. Grosskreutz, H., Rüping, S.: On subgroup discovery in numerical domains. Data Min. Knowl. Discov. 19(2), 210-226 (2009)

16. Howard, J.D., Plailly, J., Grueschow, M., Haynes, J.D., Gottfried, J.A.: Odor quality coding and categorization in human posterior piriform cortex. Nature neuroscience 12(7), $932(2009)$

17. Kaytoue, M., Plantevit, M., Zimmermann, A., Bendimerad, A., Robardet, C.: Exceptional contextual subgraph mining. Machine Learning (2017)

18. Klösgen, W.: Explora: A multipattern and multistrategy discovery assistant. In: Advances in Knowledge Discovery and Data Mining, pp. 249-271. AAAI (1996)

19. Lavrač, N., Kavšek, B., Flach, P., Todorovski, L.: Subgroup discovery with cn2-sd. Journal of Machine Learning Research 5(Feb), 153-188 (2004)

20. van Leeuwen, M., Knobbe, A.J.: Diverse subgroup set discovery. Data Min. Knowl. Discov. 25(2), 208-242 (2012)

21. Leman, D., Feelders, A., Knobbe, A.: Exceptional model mining. In: ECMLPKDD. pp. 1-16. Springer (2008)

22. Lemmerich, F., Atzmueller, M., Puppe, F.: Fast exhaustive subgroup discovery with numerical target concepts. Data Min. Knowl. Discov. 30(3), 711-762 (2016)

23. Muthukumaraswamy, S.D., Edden, R.A., Jones, D.K., Swettenham, J.B., Singh, K.D.: Resting gaba concentration predicts peak gamma frequency and fmri amplitude in response to visual stimulation in humans. Proceedings of the National Academy of Sciences 106(20), 8356-8361 (2009)

24. de Sá, C.R., Duivesteijn, W., Soares, C., Knobbe, A.J.: Exceptional preferences mining. In: DS. pp. 3-18 (2016)

25. Schofield, P.W., Ebrahimi, H., Jones, A.L., Bateman, G.A., Murray, S.R.: An olfactory 'stress test'may detect preclinical alzheimer's disease. BMC neurology 12(1), $24(2012)$

26. Stevenson, R.J.: An initial evaluation of the functions of human olfaction. Chemical senses 35(1), 3-20 (2009)

27. Tukey, J.W.: Exploratory data analysis, vol. 2. Reading, Mass. (1977)

28. Wrobel, S.: An algorithm for multi-relational discovery of subgroups. In: PKDD. pp. 78-87. Springer (1997) 